\title{
Challenge to front-loading of the vehicle development using virtual prototyping and reformation of the process
}

Atsushi Tsubouchi, Honda R\&D Co., Ltd. Automobile R\&D Center, Japan

This manuscript is not available according to publishing restriction. Thank you for your understanding. 